\title{
Research Paper: Comparing the Diadochokinetic Rate in Farsi-Speaking Young and Older Adults
}

\author{
Seyyede Zohre Mousavi ${ }^{1}$ (D), Azar Mehri ${ }^{*}$, Delaram Nabavi² (iD, Marziyyeh Faraji ${ }^{3}$ (D), Saman Maroufizadeh ${ }^{4}$ (D) \\ 1. Department of Speech Therapy, School of Rehabilitation Sciences, Iran University of Medical Sciences, Tehran, Iran. \\ 2. Department of Speech Therapy, School of Rehabilitation, Tehran University of Medical Sciences, Tehran, Iran. \\ 3. Department of Speech Therapy, University of Welfare and Rehabilitation Sciences, Tehran, Iran \\ 4. School of Nursing and Midwifery, Guilan University of Medical Sciences, Rasht, Iran
}

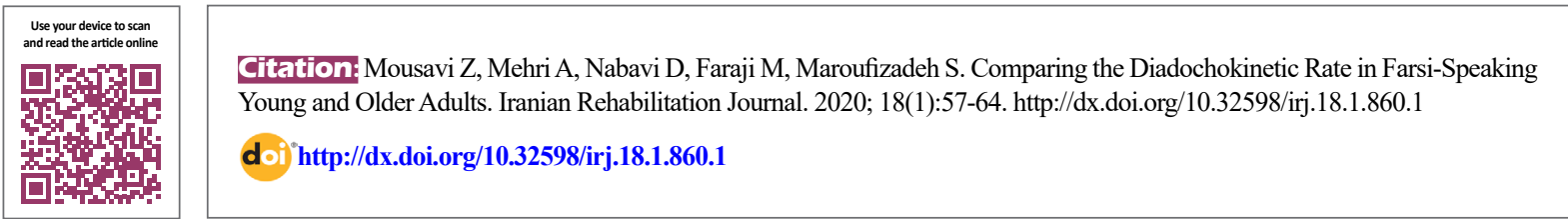

Article info:

Received: 10 Jam2019

Accepted: 05 Oct 2019

Available Online: 01 Mar 2020

Keywords:

Diadochokinetic, Elderly, Young, Persian adults

\section{A B S T RACT}

Objectives: Speech Diadochokinesis (DDK) is defined as the ability to produce repetitive and fast speech movements. This study aimed at comparing the DDK rate between Farsi-speaking young and older adults.

Methods: In this study, 244 normal adults (122 males and 122 females) were divided into two groups of young people (20-49 years) and elderly (50-69 years). DDK tasks was performed based on two ways: the participant was asked to produce /pa//ta///ka//pata//,paka//taka/, and /pataka/ sounds in $5 \mathrm{~s}$; and to repeat single syllable sounds $(/ \mathrm{pa} / / \mathrm{ta} / / \mathrm{ka} /) 20$ times, two syllables sounds (/pata//paka//taka/) 15 times, and a three syllables sound (/pataka/) ten times, separately and quickly. The independent T-test, one-way ANOVA, and Pearson correlation were used for data analysis.

Results: The results indicated a significant negative and weak correlation between age and all syllables in the specified time (range: -0.139 to -0.254 ), and a positive and weak correlation between age and time with respect to the repetition of all syllables (range: 0.121 to 0.245 ). There was no significant difference between genders in both ways $(\mathrm{P}>0.05)$.

Discussion: The results indicated different DDK rate between the young and elderly subjects that can be used as a benchmark for the diagnosis of disabled individuals in producing repetitive and fast oral movements and also as a basis for clinical assessments.

\section{* Corresponding Author:}




\section{Highlights}

- Our results found a criterion for assessing the ability to produce oral movements in normal young and elderly subjects.

- It is suggested that education is effective on the DDK rate; however, gender was not found effective.

\section{Plain Language Summary}

Oral diadochokinesis rate refers to the ability to produce fast and repetitive movements with vocal organs. The DDK rate can indicate the physiological and structural changes in the central nervous system and the peripheral components of the oral and speech production mechanisms. Therefore, the aim of this study was to compare the DDK rate between Iranian young adults and elderly. The results indicated that the elderly's diadochokinesis rate was slower than the young people, which can be used for the diagnosis of individuals unable to produce repetitive and fast oral movements and also as a basis for clinical assessments.

\section{Introduction}

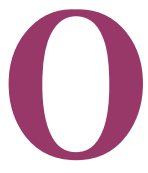

ral diadochokinesis (DDK) refers to the ability to produce fast and repetitive movements with vocal organs [1]. Fletcher believed that in terms of physiological skills, DDK is important because can represent free movements as a repeatable activity with respect to muscle balance. This means that a subtle balance should be maintained between the muscle tone, which is necessary for maintaining a fixed state. Therefore, the autonomous and integrated functioning of the muscles requires alternating movements. The time required for each movement is measurable and is a quantitative reflection of the muscle's normal and pathological function. DDK rate has been considered by many speech and language pathologists since it can be studied by measuring the relationship between motor ability and speech production. The DDK rate in a person's speech depicts his ability to express different movements (or repetitions) in the puh-tuh-kuh syllables [1].

The Alternating Motion Rate (AMR) and Sequential Motion Rate (SMR) tasks are two forms of the DDK test, which are usually used to evaluate speech movements [2] and are suitable for assessing the variety and intensity of speech disorders in people with different levels of linguistic and cognitive abilities [3]. The DDK rate has traditionally been used to evaluate, diagnose, and treat patients with oral cancer, glossectomy, musculoskeletal disorders, swallowing disorder, functional speech disorders, and speech apraxia. The DDK rate can indicate the physiological and structural changes in the central nervous system and the peripheral components of the oral and speech production mechanisms [4].
In particular, for all types of speech and language disorders and swallowing difficulty in the dysarthria, a detailed oral-peripheral examination of the resting skeletal muscle should be considered. Hence, a speech and language pathologist evaluates the chewing muscles by observing its structure, symmetry, force, accuracy, and speed [5].

According to the United States Census Bureau in 2003, there are about 35.5 million elderly in the United States [5] and based on the 2007 census in Iran, more than 6.5 million people are 60 years old and over, accounting for $8.2 \%$ of the Iran's total population. In 2006, this proportion was recorded as $7.3 \%$ [6]. This figure indicates the growth of the elderly population in Iran. Accordingly, addressing the communication and speech issues in this population is necessary due to the prevalence of speech disorders, such as swallowing, vocal, and motor problems, like dysarthria and Parkinson Disease (PD). Some studies have been conducted on DDK speed in Iranian children and adolescents [7, 8]. Although their results can be used as a reliable scale for speech therapists; however, no comprehensive study has been conducted in adults. Therefore, the present study was designed to evaluate and compare the rate of DDK in both young and older adults.

DDK has been affected by several speech and language problems in adults, for example, in patients with traumatic brain injury [3, 9], PD [10], and also in adults with hearing impairment [11]; however, a few studies have examined and compared the speed of DDK in older adults and young people. Pierce et al. studied the AMR and SMR in the elderly population using acoustic analysis software and examined the effects of age and gender [2]. The results of a study on DDK rate in young and el- 
derly subjects showed that younger people had a higher DDK rate in all tasks than the elderly [12].

Ashley et al. also addressed the speech, language, and swallowing disorders in the elderly. The DDK tasks can be used to assess the speed, accuracy, and control of the rhythm of phonation, respiration, and production [5]. Prathanee studied the DDK rate in adults and reported that the results of his study could be used as a guide for studying the DDK rate in normal people [4]. Parnell and Amerman surveyed oral DDK among the young, older, and dysarthric patients. They emphasized the need for raising the clinical standards for defining the normative rate for the elderly population [13], and Fletcher provided the normal DDK rate data for adults [14].

The purpose of this study was to compare the ability of Farsi-speaking young and older adults regarding their motor abilities (DDK rate) using two methods as mentioned above. We also aimed at assessing the effects of gender and education on DDK rate. The obtained data can be used as a benchmark by examining the rate of fast and alternating movements of the vocal organs in the normal young and elderly subjects.

\section{Methods}

This descriptive-analytic study was conducted on normal young adults (20-49 years old) and the elderly (50-69 years old) including both genders in the general places in Tehran, such as universities, offices, educational classes, libraries, and parks using cross-sectional and non-random sampling. The young people were divided into three age groups of 20-29, 30-39, and 40-49 years old and the elderly into two age groups of 50-59 and 60-69 years old. After completing the informed consent forms, the personal information of the participants in different age groups was recorded and assessed according to the inclusion criteria of the study.

The inclusion criteria included: 1) no history of head injury, tumor, epilepsy, stroke, and other neurological injuries; 2) no other psychiatric disorders; 3) no history of drug and alcohol abuse; and 4) no history of sensory impairments, such as hearing and vision problems or lose. Then, the experiment instructions were explained to the subjects according to the two methods for performing the DDK assessment. In the first method, the individuals were asked to repeat the $/ \mathrm{pa} /, / \mathrm{ta} /, / \mathrm{ka} /$, then $/ \mathrm{paka} /, / \mathrm{taka} /$, and /pata/ followed by the /pataka/ syllables as fast as they could within $5 \mathrm{~s}$. Their voices were recorded using a MP3 player for later analysis.
In the second method, the subjects were asked to repeat 20 repetitions of the single syllables (/ka/, /ta/, /pa/), 15 repetitions of the two syllables (/paka/, /taka/, and / pata/) and 10 repetitions of the three syllables (/pataka/) separately and quickly. At the same time, the examiner recorded the time spent for producing the syllables and their voices once again. In both methods, the examiner first performed them for the subjects and allowed him/ her to practice them once before performing and recording the sound.

To determine the duration and number of the repetitive syllables accurately, the recorded sounds were transmitted to the Praat software v. 5.3.52 and analyzed. The DDK rate was measured to determine the acoustic index of the syllables per second using the mean peak number or peak intensity variation.

Descriptive and inferential statistics were used for data analysis. Descriptive statistics were used to prepare the tables, plots, and calculate the statistical indices. Inferential statistics, such as the independent t-test, one-way ANOVA (Tukey post-hoc test), and Pearson correlation coefficient were used for studying the research objectives. The data were analyzed through SPSS software V. 16 at the significance level of 0.05 .

\section{Results}

A total of 244 subjects (122 males and 122 females) participated in this study and were divided into two groups of the young people and the elderly. The mean age of the participants was $43.91 \pm 14.41$ years. Among the participants, 101 cases $(41.4 \%)$ had completed an undergraduate degree and higher education and 72 cases (29.5\%) had governmental jobs. The average number of syllables spoken within the $5 \mathrm{~s}$ is presented in Table 1. The average time spent to express the number of syllables according to the age group and gender is presented in Table 2.

The Pearson correlation coefficient was used to determine the relationship between age and number of syllables spoken within $5 \mathrm{~s}$. The results indicated a negative and significant, but poor correlation between age and all spoken syllables (range: -0.139 to -0.254 ) (Table 3 ). In addition, the results indicated a positive and significant, but poor correlation between the age and time spent for all the syllables to be repeated, except for the /pata/ syllable (range: 121.12 to 0.244 ) (Table 4).

The one-way ANOVA test was used to compare the mean number of syllables repeated after classifying the subjects in five age groups (20-69 years). As shown in 
Table 1. The average number of syllables expressed in $5 \mathrm{~s}$ by age and gender $(n=244)$

\begin{tabular}{|c|c|c|c|c|c|c|c|c|c|c|}
\hline \multicolumn{7}{|c|}{ Young Adults } & \multicolumn{4}{|c|}{ Older Adults } \\
\hline \multirow{2}{*}{$\begin{array}{l}\text { Age (y) } \\
\text { Gender }\end{array}$} & \multicolumn{2}{|c|}{$20-29$} & \multicolumn{2}{|c|}{$30-39$} & \multicolumn{2}{|c|}{$40-49$} & \multicolumn{2}{|c|}{$50-59$} & \multicolumn{2}{|c|}{$60-69$} \\
\hline & M & $\mathbf{F}$ & M & $\mathbf{F}$ & M & $\mathbf{F}$ & M & $\mathbf{F}$ & M & $\mathbf{F}$ \\
\hline / Pa / & 26.07 & 26.08 & 27.70 & 25.42 & 24.37 & 25.20 & 23.41 & 24.87 & 22.54 & 23.04 \\
\hline / Ta / & 25.65 & 25.25 & 27.12 & 25.26 & 24.12 & 25.29 & 22.91 & 24.25 & 22.29 & 21.00 \\
\hline / Ka / & 23.76 & 23.75 & 26.00 & 23.19 & 22.95 & 23.41 & 21.33 & 24.12 & 29.87 & 20.29 \\
\hline / Pata / & 14.73 & 14.70 & 16.04 & 14.65 & 14.08 & 14.37 & 13.58 & 14.29 & 14.50 & 13.12 \\
\hline / Taka / & 13.73 & 13.62 & 14.66 & 13.65 & 13.12 & 13.37 & 12.62 & 13.12 & 13.20 & 12.58 \\
\hline / Paka / & 14.23 & 14.37 & 15.25 & 14.07 & 13.79 & 13.87 & 12.91 & 14.04 & 13.33 & 12.95 \\
\hline /Pataka / & 10.80 & 10.91 & 11.08 & 10.73 & 10.79 & 10.29 & 9.62 & 10.58 & 9.45 & 9.83 \\
\hline
\end{tabular}

Iranian Rehabilitation Journal

Figure 1, there was a significant difference among the age groups regarding the number of syllables of $/ \mathrm{pa} /, / \mathrm{ta} /$, $/ \mathrm{ka} /$, and /pataka/ $(\mathrm{P}<0.05)$. The Tukey's post-hoc test results indicated that the average number of syllables, including $/ \mathrm{pa} /, / \mathrm{ta} /, / \mathrm{ka} /$, and $/$ pataka/ in the age groups 20-29 and 30-39 years were significantly higher than the age group of 60-69 years $(\mathrm{P}<0.05)$; however, in the older age groups, the average number of syllables repeated, including /pata/, /taka/, and/paka/ decreased, but this difference was not statistically significant $(\mathrm{P}>0.05)$.
In addition, there was a significant difference among the age groups in terms of the time spent for 20 repetitions of $/ \mathrm{pa} /, / \mathrm{ta} /$, and $/ \mathrm{ka} /$ and 10 repetitions of /pataka/ $(\mathrm{P}<0.05)$. The Tukey's post-hoc test results indicated that the mean time needed for 20 repetitions of $/ \mathrm{pa} /, / \mathrm{ta} /$, and $/ \mathrm{ka} /$ and 10 repetitions of /pataka/ in the age groups of 20-29 and 30-39 years were significantly lower the age group of 60-69 years old $(\mathrm{P}<0.05)$. In addition, in the older age groups, the time spent for 15 repetitions of /

Table 2. The average time spent to express the number of syllables by age and gender $(n=244)$

\begin{tabular}{|c|c|c|c|c|c|c|c|c|c|c|c|}
\hline \multirow{3}{*}{ No. } & \multicolumn{7}{|c|}{ Young Adults } & \multicolumn{4}{|c|}{ Older Adults } \\
\hline & \multirow{2}{*}{$\begin{array}{l}\text { Age (y) } \\
\text { Gender }\end{array}$} & \multicolumn{2}{|c|}{$20-29$} & \multicolumn{2}{|c|}{$30-39$} & \multicolumn{2}{|c|}{$40-49$} & \multicolumn{2}{|c|}{$50-59$} & \multicolumn{2}{|c|}{$60-69$} \\
\hline & & $M$ & $\mathbf{F}$ & M & $\mathbf{F}$ & M & $\mathbf{F}$ & M & $\mathbf{F}$ & M & $\mathbf{F}$ \\
\hline \multirow{3}{*}{20} & / Pa / & 4.02 & 3.94 & 3.67 & 4.09 & 4.30 & 4.31 & 4.44 & 4.25 & 4.69 & 4.55 \\
\hline & / Ta / & 4.12 & 4.08 & 3.85 & 4.24 & 4.44 & 4.43 & 4.74 & 4.48 & 4.77 & 5.05 \\
\hline & / Ka / & 4.40 & 4.33 & 4.10 & 4.70 & 4.59 & 4.70 & 5.02 & 4.51 & 4.99 & 5.29 \\
\hline \multirow{3}{*}{15} & / Pata / & 5.33 & 5.16 & 4.76 & 5.37 & 5.69 & 5.51 & 5.71 & 5.23 & 5.28 & 5.93 \\
\hline & / Taka / & 5.62 & 5.63 & 5.30 & 5.68 & 5.91 & 6.03 & 6.12 & 5.78 & 5.72 & 6.15 \\
\hline & / Paka / & 5.52 & 5.30 & 5.10 & 5.54 & 5.71 & 5.76 & 6.07 & 5.55 & 5.65 & 5.92 \\
\hline 10 & /Pataka / & 4.76 & 4.74 & 4.60 & 4.74 & 4.88 & 5.05 & 5.35 & 5.00 & 5.27 & 5.38 \\
\hline
\end{tabular}

Iranian Rehabilitation Journa

Table 3. Correlation analysis between age and number of syllables spoken in $5 \mathrm{~s}(\mathrm{n}=244)$

\begin{tabular}{ccc}
\hline Syllables & Pearson Coefficient & P \\
\hline$/ \mathrm{pa} /$ & -0.224 & $<0.001$ \\
\hline$/ \mathrm{ta} /$ & -0.229 & $<0.001$ \\
\hline$/ \mathrm{ka} /$ & -0.201 & 0.002 \\
\hline /pata/ & -0.139 & 0.030 \\
\hline /taka/ & -0.157 & 0.014 \\
\hline /paka/ & -0.165 & 0.010 \\
\hline /pataka/ & -0.254 & $<0.001$ \\
\hline
\end{tabular}


Table 4. Correlation analysis between age and time spent to repeat syllables $(n=244)$

\begin{tabular}{ccc}
\hline Time & Pearson Coefficient & P \\
\hline 20 repeations of $/ \mathrm{pa} /$ & 0.221 & 0.001 \\
\hline 20 repeations of $/ \mathrm{ta} /$ & 0.245 & $<0.001$ \\
\hline 20 repeations of $/ \mathrm{ka} /$ & 0.212 & 0.001 \\
\hline 15 repeations of $/ \mathrm{pata} /$ & 0.121 & 0.059 \\
15 repeations of $/ \mathrm{taka} /$ & 0.135 & 0.034 \\
\hline 15 repeations of $/ \mathrm{paka} /$ & 0.130 & 0.043 \\
\hline 10 repeations of $/ \mathrm{pataka} /$ & 0.219 & 0.001 \\
\hline
\end{tabular}

Iranian Rehabilitation \ourna

pata/, /taka/, and /paka/ syllables increased; however, it was not statistically significant $(\mathrm{P}>0.05)$.

Based on the results of the independent t-test, there was no significant difference regarding the mean number of syllables repeated between women and men $(\mathrm{P}>0.05)$. In addition, no statistically significant difference was found between both genders in the time spent for repeating the syllables $(\mathrm{P}>0.05)$.

There was a significant difference between the education level of the participants and the number of syllables, including $/ \mathrm{ta} /, \mathrm{ka} /$, and $/$ pataka/ $(\mathrm{P}<0.05)$. The results of Tukey's post-hoc test indicated that the average number of syllables, such as /ta/ and /pataka in the cases with bachelor's and higher education degrees was significantly higher than those with high school education $(\mathrm{P}<0.05)$. In addition, the average number of $/ \mathrm{ka} /$ syllables repeated in the group with higher education degree was significantly higher than those with high school education $(\mathrm{P}<0.05)$. Moreover, as the educational levels increased, the average number of repeated syllables, such as /pa/, /pata/,/taka/, and /paka/ increased; however, it was not statistically significant $(\mathrm{P}>0.05)$.
There was a significant difference between the subjects' education level regarding the time spent for 20 repetitions of the syllables $/ \mathrm{ta} / \mathrm{and} / \mathrm{ka} /$ and 10 repetitions of /pataka/ $(\mathrm{P}<0.05)$. The average time required for 20 repetitions of the syllables $/ \mathrm{ta} / \mathrm{and} / \mathrm{ka} /$ in the group with higher education was significantly lower than those with high school education $(\mathrm{P}<0.05)$. In addition, the average time needed for 10 repetitions of the syllable /pataka/ in the cases with bachelor's and higher education degrees was significantly lower than those with high school education $(\mathrm{P}<0.05)$. Moreover, as the education level increased, the time spent for 20 repetitions of the syllable /pa/ and 15 repetitions of /pata/, /taka/, and /paka/ decreased, however, this difference was not statistically significant $(\mathrm{P}>0.05)$.

\section{Discussion}

An increase in the population of older people in Iran along with the high prevalence of communication disorders, such as swallowing difficulties and voice and motor disorders, such as dysarthria and PD indicate that motor speech disorders should be considered. As mentioned previously, several studies have examined the DDK

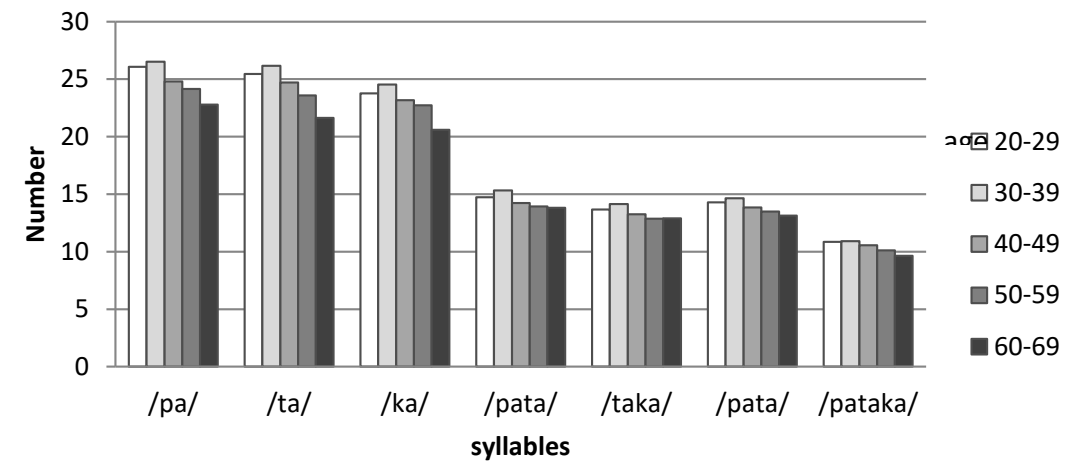

Figure 1. Comparing the number of studied syllables in $5 \mathrm{~s}$ according to the age group 
speed in children and people having different disorders; however, very few studies have addressed this issue in normal adults and older people.

The aim of this study was to compare the rate of DDK in young and older people to find a criterion for assessing the ability to produce fast and repetitive speech movements in normal adults. The obtained findings can be used to identify people with these kinds of disabilities and are helpful in designing an appropriate treatment program for the affected groups.

The results of this study demonstrated that the average number of syllables repeated, such as $/ \mathrm{pa} /, / \mathrm{ta} /, / \mathrm{ka} /$, and /pataka/ in the age groups of 20-29 and 30-39 years was significantly higher than the age group of 60-69 years. In addition, with an increase in age, the average number of repeated /pata/, /taka/, and /paka/syllables decreased; however, these differences were not statistically significant. Regarding the production of syllables within the 5 $\mathrm{s}$, the younger subjects performed better than the elderly. This finding is in line with the results reported by $\mathrm{Pa}$ dovani et al. They examined the DDK rate in 23 adults aged 30-46 and 23 adults aged 47-94 years old using the using the Visi-Pitch III/Sona-Speech (Kay Elemetrics, Inc.) voice analysis software.

The results indicated that younger subjects had higher DDK rates [12]. In addition, in a review by Ashley et al. on speech, language, and swallowing disorders in elderly, the authors stated that the elderly patients comprise one of the most commonly referred patients to the speech and language pathologists. In addition, they also reported that the DDK tests can assess the speed, accuracy, and control of the rhythm of phonation, breathing, and production. These studies have demonstrated the problems faced by elderly in performing tasks, in which the speed and accuracy of motion are involved as discussed in the present study [5].

The findings of other studies are in line with our results. In 1987, Parnell and Amerman asked two speech pathologists to judge the adequacy of oral DDK in 10 men, aged 21-28 years, 10 geriatric men aged 67-81 years, and four dysarthric patients. The criterion 1 indicates normality and the criterion 7 indicates a severe deviation from normality. The results indicated significant differences among the three groups, wherein the elderly subjects were found at the end of the normal range and close to the dysarthric patients [13].

Therefore, the results of the older population were different from the younger population; however, their results were not consistent with the results of the study by Pierce et al. They studied the AMR for the syllables /pa/, /ta/, and / ka/ and the SMR for the syllable /pataka/ in adults aged 65 years and older. The rate of repetition of the syllables for each task was measured using acoustic analysis software and the effects of age and gender were studied. The results indicated that age had no significant effect on the tasks, which can be due to the studied age group (those over 65 years old) as it is suggested that elderly subjects often suffer from speech, language, and swallowing problems [2].

The average time required for 20 repetitions of the syllables $/ \mathrm{pa} /, / \mathrm{ta} /$, and $/ \mathrm{ka} /$ and 10 repetitions of the syllable /pataka/ in the age groups of 20-29 and 30-39 years was significantly lower than the age group of 60 69 years old. In addition, the duration of 15 repetitions of the syllables /pata/, /taka/, and /paka/ increases by an increase in the age of the subjects, however, these differences were not statistically significant. This issue can be attributed to a weakness of the muscles in the organs participating in speech in the elderly subjects, which can reduce the number of syllables at a specified time and increase the time needed for producing the syllables. In general, the non-significant results can be due to the age of 69 years as this age is the beginning of age-related and neuromuscular problems in the elderly. Significant results can possibly be obtained by studying those aged over 70 years old.

Our results indicated a negative and significant, but the poor correlation between age and all syllables repeated within $5 \mathrm{~s}$; in other words, the number of syllables produced decreased with an increase in the age of the participants. In addition, there was a positive and significant, but poor correlation between age and the time needed to repeat all syllables (except for/pata/), and also the time required to repeat the specified syllables was increased with increasing age.

The findings indicated no significant difference between women and men regarding both methods (fixed time and number of syllables) of evaluating the DDK rate. This suggests that the DDK performance was more influenced by age than gender; therefore, it was not an effective factor. The only study that reported genderrelated findings was conducted by Pierce et al. (2013); however, their findings were not consistent with our results. They reported gender as an effective factor in the rate of speech motion and men significantly performed better than women [2]. 
There was a significant difference between the education and number of repetition of $/ \mathrm{ta} /, / \mathrm{ka} /$, and /pataka/ syllables. In addition, the average number of produced syllables, including /pa/, /pata/, /taka/, and /paka/ increased with increasing educational levels; however, these differences were not statistically significant. Education has shown effective on the DDK rate. The elderly subjects with lower educational level had a lower DDK rate than younger cases as expected. Similarly, regarding the time needed to repeat the syllables, those with higher education required a shorter time to repeat syllables (except for $/ \mathrm{ta} /, / \mathrm{ka} /$, and /pataka/ syllables). It should be noted that the effect of education on DDK has not yet been studied.

The obtained findings can be used to assess the DDK rate in different age ranges in the normal population and also in clinical settings as a benchmark for measuring the speed of oral movements in several neurological diseases because these patients face difficulties in making oral movements. In this regard, the results of a study conducted in England have long been used as a benchmark for comparing the individuals with speech disorders [14]. One of the advantages of this method is that it cannot be affected by a cognitive impairment in patients; therefore, it has been used for disorders with all levels of severity. In addition, in Iran there are numerous spoken dialects which vary from province to province that is not associated with cognitive impairment, therefore, the obtained DDK rate can be used in all clinical settings as a benchmark with other assignments that measure motor skills. Also, our findings can provide comprehensive data for future studies to assess auditory, voice, and motor disorders. One of the limitations of this study was the poor cooperation of some nursing centers for sampling the elderly subjects.

\section{Conclusion}

The obtained data can be used as a benchmark for identifying the people with impaired fast and alternating oral movements and should be useful for planning the most appropriate treatment programs according to the severity of the disorder.

\section{Ethical Considerations}

\section{Compliance with ethical guidelines}

This study was approved by the Ethics Committee of the Tehran University of Medical Science and the Rehabilitation Research Center for Sensory-Motor Disorders (Code: IR.TUMS.REC.1395.2665). Also, the written informed consent was obtained from the participants.

\section{Funding}

This study was funded by the Rehabilitation Research Center for Sensory-Motor Disorders, Tehran University of Medical Sciences.

\section{Authors' contributions}

All authors contributed in designing, running, and writing all parts of the research.

\section{Conflict of interest}

The authors declared no conflict of interests.

\section{Acknowledgments}

We thank the center's managers and all participants who participated in this research.

\section{References}

[1] Nicolosi L, Harryman E, Kresheck J. Terminology of communication disorders: Speech-language-hearing. Philadelphia: Lippincott Williams \& Wilkins; 2004.

[2] Pierce JE, Cotton S, Perry A. Alternating and sequential motion rates in older adults. International Journal of Language \& Communication Disorders. 2013; 48(3):257-64 [DOI:10.1111/1460-6984.12001] [PMID]

[3] Wang Yt, Kent RD, Duffy JR, Thomas JE, Weismer G. Alternating motion rate as an index of speech motor disorder in traumatic brain injury. Clinical Linguistics \& Phonetics. 2004; 18(1):57-84. [DOI:10.1080/02699200310001596160] [PMID]

[4] Prathanee B. Oral diadochokinetic rate in adults. Journal of the Medical Association of Thailand. 1998; 81(10):784-8.

[5] Ashley J, Duggan M, Sutcliffe N. Speech, language, and swallowing disorders in the older adult. Clinics in Geriatric Medicine. 2006; 22(2):291-310. [DOI:10.1016/j.cger.2005.12.008] [PMID]

[6] Mirzaei M, Shams-Ghahfarkhi M. Demographic characteristics of the elderly population in Iran according to the census 1976-2006. Salmand: Iranian Journal of Ageing. 2007; 2(5):32631.

[7] Shahbodaghi MR, Moradi A, Kiani S. [Diadochokinetic syllable rates standardized among the elementary students (Persian)]. Bimonthly Audiology. 2005; 14(1):39-49.

[8] Ahadi HSM, Shahbodaghi MR, Mokhlesin M, MahamoudiBakhtiari B. [A comparative study of diadochokinesis and the rate of reading and speech in second grade primary school students of Tehran, Iran (Persian)]. Audioral. 2009; 18(1-2):63-9. 
[9] Blumberger J, Sullivan SJ, Clement N. Diadochokinetic rate in persons with traumatic brain injury. Brain injury. 1995; 9(8):797-804. [DOI:10.3109/02699059509008235] [PMID]

[10] Ackermann H, Konczak J, Hertrich I. The temporal control of repetitive articulatory movements in Parkinson's disease. Brain and Language. 1997; 56(2):312-9. [DOI:10.1006/ brln.1997.1851] [PMID]

[11] Robb MP, Hughes MC, Frese DJ. Oral diadochokinesis in hearing-impaired adolescents. Journal of Communication Disorders. 1985; 18(2):79-89. [DOI:10.1016/0021-9924(85)90038-3]

[12] Padovani M, Gielow I, Behlau M. Phonarticulatory diadochokinesis in young and elderly individuals. Arquivos de Neuro-psiquiatria. 2009; 67(1):58-61. [DOI:10.1590/S0004282X2009000100015] [PMID]

[13] Parnell MM, Amerman JD. Perception of oral diadochokinetic performances in elderly adults. Journal of Communication Disorders. 1987; 20(4):339-51. [DOI:10.1016/00219924(87)90015-3]

[14] Fletcher SG. Time-by-count measurement of diadochokinetic syllable rate. Journal of Speech and Hearing Research. 1972; 15(4):763-70. [DOI:10.1044/jshr.1504.763] [PMID] 